\title{
Exploring the deep convection and magnetism of A-type stars
}

\author{
Nicholas A. Featherstone ${ }^{1}$, Matthew K. Browning ${ }^{2}$, \\ Allan Sacha Brun ${ }^{3}$ and Juri Toomre ${ }^{1}$ \\ ${ }^{1}$ JILA and Department of Astrophysical and Planetary Sciences, University of Colorado, \\ Boulder, CO 80309-0440, USA \\ email:feathern@lcd.colorado.edu \\ ${ }^{2}$ Canadian Institute for Theoretical Astrophysics, University of Toronto, Toronto, \\ ON M5S3H8, Canada \\ ${ }^{3}$ DSM/IRFU/SAp, CEA-Saclay and UMR AIM, CEA-CNRS-Universite Paris 7, \\ 91191 Gif-sur-Yvette, France
}

\begin{abstract}
A-type stars have both a near-surface layer of fast convection that can excite acoustic modes and a deep zone of core convection whose properties may be probed with asteroseismology. Many A-type stars also exhibit large magnetic spots that are often attributed to surviving primordial fields of global scale in the intervening radiative zone. We have explored the potential for core convection in rotating A-type stars to build strong magnetic fields through dynamo action. These 3-D simulations using the ASH code provide guidance on the nature of differential rotation and magnetic fields that may be present in the deep interiors of these stars, thus informing the asteroseismic deductions now becoming feasible. Our models encompass the inner $30 \%$ by radius of a two solar mass A-type star, rotating at four times the solar rate and capturing the convective core and a portion of the overlying radiative envelope. Convection in these stars drives a strong retrograde differential rotation and yields a core that is prolate in shape. When dynamo action is admitted, the convection generates strong magnetic fields largely in equipartition with the dynamics. Remarkably, introducing a modest but large-scale external field threading the radiative envelope (which may be of primordial origin) can substantially alter the turbulent dynamics of the convective interior. The resulting convection involves a complex assembly of helical rolls that link distant portions of the core and stretch and advect magnetic field, ultimately yielding magnetic fields of super-equipartition strength.
\end{abstract}

Keywords. Astroseismology, A-type stars, differential rotation, deep convection

\section{Introduction}

The peculiar A stars (Ap) exhibit strong and variable spectral lines (relative to solar values) in $\mathrm{Si}$ and certain rare earth metals (e.g., $\mathrm{Sr}$ and $\mathrm{Hg}$ ). Of the $\mathrm{Si}$ and $\mathrm{Sr}-\mathrm{Cr}-\mathrm{Eu}$ peculiarity classes, many possess equally variable and unusually strong magnetic fields with strengths ranging from a lower threshold of 300 G up to 20,000 G (e.g., Mestel 1999; Aurière et al. 2007). When variable, these fields appear to change at the stellar rotation rate (e.g., Deutsch 1956; Mestel 1999), suggesting that the magnetic fields are "frozen in" to the radiative envelope of the star. A typical Ohmic decay time is much longer than the lifetime of an A star, and so these magnetic fields are likely the remnants of a primordial magnetic that threaded the star's natal molecular cloud (Cowling 1945).

The subsurface nature of these strong fields remains unknown and may depend on the dynamics of the star's convective core. Recently, 3-D numerical simulations have demonstrated that nuclear burning cores of A-type stars can drive vigorous convection capable of generating equipartition strength magnetic fields (Browning et al. 2004; Brun 


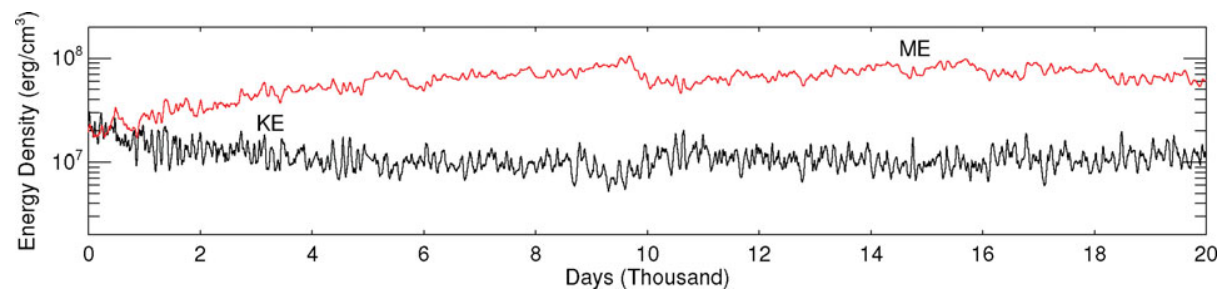

Figure 1. Evolution of volume-averaged energy densities following the imposition of an external mixed magnetic field spanning 20,000 days (or about 2,900 rotation periods). Magnetic energy $\mathrm{ME}$ (red) has grown in strength to become about ten-fold greater than the kinetic energy KE (black) and is thus super-equipartition in nature.

et al. 2005). If the surface fields are indicative of a global-scale magnetic field with roots in the core, then the primordial field must exert some influence on the core dynamo, and vice versa. We explore this possibility by modeling a core dynamo with a large-scale field of primordial origin threading the radiative envelope.

\section{Modeling the A Star}

We use the 3-D anelastic spherical harmonic (ASH) code (see Brun et al. 2004) to model convection and dynamo action in a main-sequence A-type star of $2 M_{\odot}$ rotating at four times the solar rate (with a rotation period of seven days). Our computational domain encompasses the inner 0.3 of the A star by radius, the inner 0.15 of which is convectively unstable, with an overlying stable, radiative envelope comprising the remainder of the domain. We adopt a Prandtl number of 0.25 and a magnetic Prandtl number of 5 throughout the domain, achieving a Reynolds number following equilibration of $\sim 136$ and a magnetic Reynolds number of $\sim 680$. Further details of this model may be found in Featherstone et al. (2009).

Our fossil field system was initialized by augmenting the magnetic fields from a mature A-star dynamo simulation (case C4m) of Brun et al. (2005). Their dynamo was started using a well equilibrated hydrodynamic simulation from Browning et al. (2004) by adding a small dipole seed field to this system. Persistent dynamo action realized in that simulation yielded equipartion magnetic energies with respect to the convective flows.

The geometry most likely to support a primordial field against decay is likely to require a poloidal and toroidal component if the field is to remain stable over the lifetime of a star (e.g., Prendergast 1956; Braithwaite \& Spruit 2004). We have adopted such a twisted field geometry for our model fossil field, placing a magnetic torus with amplitude $30 \mathrm{kG}$ in the lower radiative zone. A poloidal field component consistent with a current threading through the center of our magnetic torus was then added to give some twist to the field, yielding a fossil field whose magnetic energy constituted a modest $10 \%$ increase to the magnetic energy of the overall system.

\section{Discussion}

The most striking effect arising from the inclusion of a fossil field in our core dynamo model is apparent in the temporal evolution of the system's (globally-averaged) energy densities. The inclusion of a fossil magnetic field in our system has led to a fivefold increase in the magnetic energy (ME) that gradually develops over approximately one magnetic diffusion time ( 7000 days) following the imposition of the external field. As 

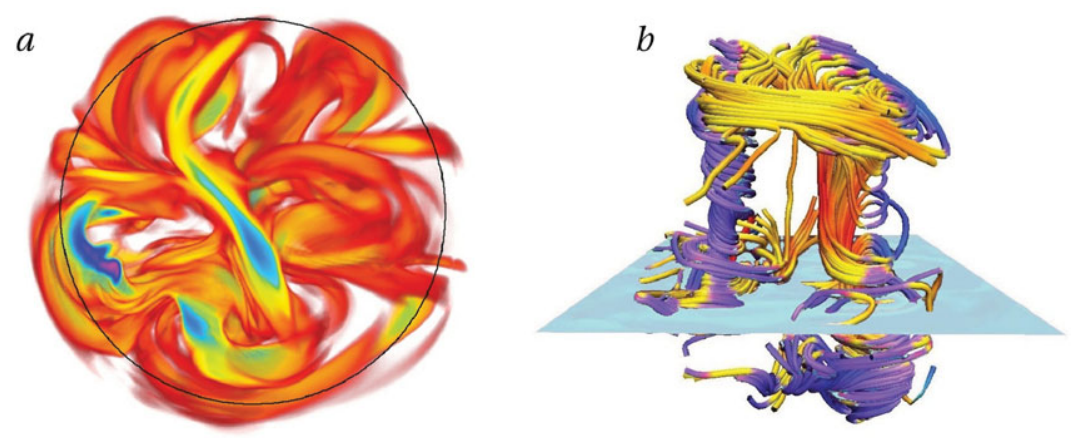

Figure 2. Convective and magnetic structures near day 15,000. (a) Magnetic magnetic energy density as realized in the equatorial region of the convection zone and region of overshooting; yellow/green tones indicate high values, and red tones low values. (b) Interior view of columnar convection in the core of the A-star visualized near day 15,000 using instantaneous streamlines. Streamlines are colored by velocity component along the rotation axis $v_{z}$. Blue (yellow) tones indicate northward (southward) motion; equatorial plane indicated by light blue.

the magnetic energy rises, the kinetic energy (KE) halves, yielding a ratio of $\mathrm{ME} / \mathrm{KE} \sim 10$ that is maintained (with some variation) throughout the remainder of the simulation. Such a change in the energy balance is remarkable given that the imposed fossil field constituted but a small perturbation to the magnetic energy of the system. Moreover, one might anticipate that strong Lorentz forces would accompany such high magnetic field strengths, ultimately suppressing the very convective motions that spur their genesis, but this does not occur.

The increase in ME is largely associated with non-axisymmetric magnetic fields contained within the core. Typical magnetic field strengths have transitioned from $\sim 67 \mathrm{kG}$ in the absence of a fossil field (based on rms values at mid-core) to $\sim 80 \mathrm{kG}$, and typical flow speeds are $\sim 20 \mathrm{~m} \mathrm{~s}^{-1}$ (vs. $\sim 29 \mathrm{~m} \mathrm{~s}^{-1}$ initially). While typical magnetic energies have not changed dramatically, the strong magnetic flux ropes that now pervade much of the core possess interior field strengths of $\sim 300 \mathrm{kG}$ (blue regions Figure $2 a$ ). These regions of highly concentrated magnetic flux are largely responsible for the increase in ME seen in Figure 1. Core convection in the presence of such strong magnetic fields is now characterized by four to six evolving cylindrical rolls encircling the rotation axis and aligned with it. Similar to previous studies of rotating spherical convection using rigid boundaries (e.g., Busse 2002), where these cylindrical rolls intersect the spherical edge of the convective core, their motions "tilt" as they conform to the edge of the convective core. This behavior is visible in detailed rendering of the streamlines in Figure $2 b$. Pressure gradients created by this tilting drive flows along the axes of these rolls, imbuing neighboring rolls with opposite senses of helicity. Poleward axial flows from one roll thus link to the equatorward axial flows of their neighbors. As these rolls drive only a weak differential rotation, downflows from one set of rolls freely cross the rotation axis undeflected, connecting distant regions of the core which would otherwise remain relatively isolated from one another.

The imprint that this cylindrical roll-like convection leaves on the magnetic field is illustrated in Figure $2 a$ showing magnetic energy in the equatorial region. The helical convection creates broad flux ropes of strong $(\sim 300 \mathrm{kG})$ field that extend from pole to pole encircling the periphery of the cylindrical rolls. Magnetic fields in the core thus exhibit a globally-connected topology reflective of the convective motions, with strong fields tending to exist on the periphery of regions of strong flow. Exceptions to this 
trend typically involve some co-alignment of the flows and fields that works to dimish the otherwise strong Lorentz forces (see Featherstone et al. 2009).

\section{Conclusions and Perspectives}

Our work suggests that the presence of a primordial field can subtly influence the dynamics of a core dynamo, yielding a stronger core dynamo characterized by globalscale magnetic fields and flow configurations, and a super-equipartition magnetic state. These results are quite different from those obtained earlier in the absence of a primordial field, and the state obtained may be one of a wide array of dynamo states accessible to the cores of these stars. In light of the strong field state obtained, it is particularly interesting to ask whether the strong mean fields generated near the edge of the convective core might become buoyant, ultimately contributing to the large star spots seen at the surface of the Ap and Bp stars.

\section{Acknowledgements}

The authors gratefully acknowledge Nicholas Nelson for presenting this work. This research is supported by NASA through Heliophysics Theory Program grants NNG05G124G and NNX08AI57G, with additional support for Featherstone through NASA GSRP program by award NNX07AP34H. The simulations were carried out with NSF PACI support of PSC, NCSA and TACC, and by NASA HEC support at Project Columbia. Browning was supported by NSF Astronomy and Astrophysics postdoctoral fellowship AST 0502413, and by a Jeffery L. Bishop fellowship at CITA. Brun was partly supported by the Programme National Soleil-Terre of CNRS/INSU (France), and by the STARS2 grant from the European Research Council.

\section{References}

Aurière, M. et al., 2007, A\&A 4751053

Braithwaite, J. \& Spruit, H. C., 2004 Nature 431819

Browning, M. K., Brun, A. S., \& Toomre J., 2004, ApJ 601512

Brun, A. S., Browning, M. K., \& Toomre, J., 2005 ApJ 629461

Brun A. S., Miesch, M. S., \& Toomre, J., 2004 ApJ 6141073

Busse, F. H., 2002 Phys. Fluids f 141301

Deutsch, A. J., 1956 PASP 6892

Featherstone, N. A., Browning, M. K., Brun, A. S., \& Toomre, J. 2009 ApJ 7051000

Mestel, L., 1999 Stellar Magnetism (Oxford: Clarendon Press)

Prendergast, K. H., 1956 ApJ 123498

Preston, G. W., 1971 PASP 83571

\section{Discussion}

STRAssmeIER: You said there's 300 kilogauss in your peak and you still don't see flux surfacing. So, what sort of field strength or fluxes would you need in order that flux ropes can surface up all the way up the real surface?

Featherstone: That is a good question. To address the first part, we see these very strong fields. They tend to be in the convective roles. The simulation is sufficiently diffusive that we would probably need much stronger fields, order of magnitude stronger field in order to get a buoyancy instability. We have some newer methods to try to reduce the diffusion in these simulations that may be more effective in promoting some sort of 
magnetic stability and buoyancy that hasn't been tried in the A-type stars. I'll show some results in my talk Thursday in similar things we have been able to get in solar-type stars.

ARLT: It looks like, let me call it a one-scale dynamo. You have the motions, and you have the fields. Would you expect or do you have a feeling on, when you drive up the resolution, that you can actually get scale separation and eventually end up with the possibility of describing it by a mean field dynamo? It's hard to say?

Featherstone: Yeah, it is hard to say. I would say that, in this particular case, I don't know that you are going to get a strong scale separation between your convective motions and your dynamo scales if you will in the core. I should say partly because the convective motions are - are - in terms of a spherical harmonic degree are somewhere in a spherical harmonic degree of 10,12 , or 20 , somewhere in this ballpark, which is very well-resolved in these simulations. So I don't know if your convective motions are going to get much smaller and your dynamo scales aren't going to get much larger, bigger.

ARLT: Then we would have an option of alpha-square-type turbulence which could give us these obliqueness of the things, but it doesn't look like this because you have revolved the motions already. So there's not much room for small-scale turbulence perhaps.

FeAtherstone: Yeah. 